\title{
AVALIAÇÃO MORFOLÓGICA DE DEPÓSITOS DE ZINCO ELETRO-OBTIDOS UTILIZANDO DIFERENTES CONCENTRAÇÕES DE GELATINA*
}

Tairine Berbert Tavares ${ }^{1}$ Laís Vieira Moyzés ${ }^{2}$ Ney Pinheiro Sampaio ${ }^{3}$ Tácia Costa Veloso 4

\section{Resumo}

A eletro-obtenção constitui em um importante processo na recuperação dos principais metais produzidos através de processos hidrometalúrgicos (i.e. $\mathrm{Cu}, \mathrm{Ni}, \mathrm{Au}, \mathrm{Co}, \mathrm{Zn}$ ), pois permite a obtenção de depósitos catódicos de elevada pureza a partir de soluções aquosas contendo o metal. O presente trabalho investigou a eletro-obtenção de zinco, a fim de avaliar a influência da gelatina, um aditivo orgânico, na eficiência de corrente e na qualidade do metal depositado no catodo de alumínio. A caracterização dos depósitos de zinco através do MEV revelou que, na presença de concentrações crescentes de gelatina até $40 \mathrm{mg} / \mathrm{L}$, houve diminuição da quantidade de poros ocasionados por evolução de hidrogênio na superfície do zinco depositado, contudo, em concentrações de $80 \mathrm{mg} / \mathrm{L}$, os poros voltaram a aparecer. Além disso, notou-se que diferentes concentrações de gelatina não alteraram a forma dos cristais de zinco, porém provocaram redução do tamanho de suas plaquetas hexagonais, à medida que a concentração de gelatina aumentou, mostrando efeito de refino de grão, em comparação à solução livre da adição de gelatina.

Palavras-chave: Eletro-obtenção; Zinco; Gelatina; Morfologia.

\section{MORPHOLOGICAL EVALUATION OF ZINC DEPOSITS OBTAINED BY ELECTROWINNING PROCESS USING DIFFERENT GELATIN CONCENTRATIONS}

\begin{abstract}
Electrowinning is an important process in the extraction of metals through hydrometallurgical technologies, once it promotes electrodeposition of metallic ions ( $\mathrm{Cu}, \mathrm{Ni}, \mathrm{Au}, \mathrm{Co}, \mathrm{Zn}$ ) from acidic solutions on the cathode to produce high purity metal. In this study, the electrowinning of zinc was evaluated to analyze the influence of gelatin addition on current efficiency and quality of metal deposited on aluminum cathode. The morphology of the zinc deposits analyzed through SEM shown that in the presence of increasing concentrations of gelatin until $40 \mathrm{mg} / \mathrm{L}$, there was a decreasing in the quantity of pores resulted from hydrogen evolution in the surface of zinc deposits. However, for $80 \mathrm{mg} / \mathrm{L}$ concentration, pores appeared again. Moreover, different gelatin concentrations did not change the zinc crystals shape, but the increasing on gelatin concentration promoted a reduction on its hexagonal platelets size, representing a grain refinement effect if compared to the gelatin free solution.
\end{abstract}

Keywords: Electrowinning; Zinc; Gelatin; Morphology.

1 Engenharia Metalúrgica, Graduanda, Estudante, Departamento de Engenharia Metalúrgica e de Materiais, Universidade Federal de Ouro Preto, Ouro Preto, Minas Gerais, Brasil.

2 Engenharia Metalúrgica, Graduada, Departamento de Engenharia Metalúrgica e de Materiais, Universidade Federal de Ouro Preto, Ouro Preto, Minas Gerais, Brasil.

3 Engenharia Metalúrgica, Mestre, Nanolab, Redemat, Universidade Federal de Ouro Preto, Ouro Preto, Minas Gerais, Brasil

4 Engenharia Metalúrgica, Doutora, Professora, Departamento de Engenharia Metalúrgica e de Materiais, Universidade Federal de Ouro Preto, Ouro Preto, Minas Gerais, Brasil. 


\section{INTRODUÇÃO}

Devido ao seu relativo baixo custo e abundância, o zinco é largamente utilizado na produção de latão, anodos de sacrifício, baterias primárias e secundárias (componentes eletrônicos), e aços galvanizados, cuja finalidade, entre outras, é a de proteger a estrutura contra corrosão e oxidação nas indústrias automobilística, de eletrodomésticos, da construção civil e em torres de energia e telefonia celular. Além disso, o zinco é insumo para os setores de vulcanização de borrachas e de fertilizantes; para as indústrias cerâmica, têxtil e cosmética; e para os segmentos alimentício e de medicamentos [1], sendo que seu consumo mundial foi de $12,9 \mathrm{Mt}$, em 2013, de acordo com o Departamento Nacional de Produção Mineral [2].

A principal tecnologia existente atualmente (2016) para a produção de zinco é o Processo de Ustulação-Lixiviação-Eletrólise (Roast-Leach-Electrolysis, RLE), presente em mais de $85 \%$ das usinas. Etapa final do processo e objeto de estudo deste trabalho, a eletro-obtenção de zinco consiste basicamente na transformação eletroquímica do zinco dissolvido em um eletrólito em zinco metálico depositado na superfície de um catodo. Este processo ocorre mediante à utilização de energia elétrica proveniente de uma fonte externa. Para tanto, ocorre a imersão de um catodo metálico e anodo "inerte" (mas condutivo) em um eletrólito contendo $\mathrm{ZnSO}_{4}, \mathrm{H}_{2} \mathrm{SO}_{4} \mathrm{e}$ $\mathrm{H}_{2} \mathrm{O}$. Aplica-se então uma diferença de potencial entre o catodo e o anodo, de modo que as semi-reações (Equação 1) e (Equação 2) e a reação global (Equação 3) envolvidas no processo podem ser observadas.

$$
\begin{aligned}
& \text { Reação anódica: } \mathrm{H}_{2} \mathrm{O}(\mathrm{I}) \rightarrow 1 / 2 \mathrm{O}_{2}(\mathrm{~g})+2 \mathrm{H}^{+}(\mathrm{aq})+2 \mathrm{e}^{-} \\
& \text {Reação catódica: } \mathrm{Zn}^{2+}(\mathrm{aq})+2 \mathrm{e}^{-} \rightarrow \mathrm{Zn}(\mathrm{s}) \\
& \text { Reação global: } \mathrm{Zn}^{2+}(\mathrm{aq})+\mathrm{H}_{2} \mathrm{O}(\mathrm{l}) \rightarrow \mathrm{Zn}(\mathrm{s})+1 / 2 \mathrm{O}_{2}(\mathrm{~g})+2 \mathrm{H}^{+}(\mathrm{aq})
\end{aligned}
$$

Na eletro-obtenção de zinco, diversos fatores podem afetar a eficiência de corrente e qualidade (pureza e resistência mecânica) dos depósitos produzidos nos catodos de alumínio ou do próprio metal a ser depositado. Há evidências de que a concentração de zinco no eletrólito, a acidez, a temperatura e a presença de aditivos, podem influenciar a eficiência do processo e modificar a morfologia e a estrutura cristalina do produto [3].

Tendo em vista que o aumento da eficiência de corrente no processo de eletroobtenção e a obtenção de catodos de boa qualidade são importantes do ponto de vista acadêmico/industrial, no presente estudo foi avaliado como a presença do aditivo gelatina influencia na eletro-obtenção de zinco, com o intuito de minimizar o consumo de energia da célula e contribuir para obtenção de um depósito de zinco uniforme e de elevada pureza.

\section{MATERIAIS E MÉTODOS}

Primeiramente, foi montada uma célula de eletrólise utilizando-se uma cuba de material plástico contendo três eletrodos, sendo um catodo feito de alumínio e dois anodos feitos de chumbo. Os eletrodos foram ligados à uma fonte de energia de marca e modelo EMG-18136. A referida fonte conta com voltímetro e amperímetro integrados através dos quais foram monitoradas a corrente e a voltagem. 


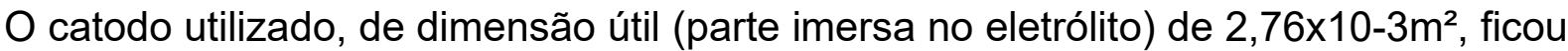
afastado dos anodos por uma distância de $1,5 \mathrm{~cm}$. Antes de cada ensaio o catodo foi polido utilizando-se lixa \#1200 e lavado.

O eletrólito contendo $157,2 \mathrm{~g} / \mathrm{L}$ de $\mathrm{Zn}$, densidade de $1,4 \mathrm{~g} / \mathrm{cm}^{3}$ e $\mathrm{pH}$ igual a 5,27 foi preparado utilizando-se ácido sulfúrico industrial. Durante os experimentos, o eletrólito foi agitado por um agitador magnético de marca e modelo Radelkis - OP-912/3 com rotação mínima. A temperatura foi monitorada por um termômetro de mercúrio graduado. Cada ensaio teve duração de cinco horas, sendo o catodo pesado antes e depois de cada ensaio para se obter a massa do depósito.

Após cada ensaio o catodo foi lavado e seco. O material depositado foi desplacado manualmente e armazenado em refrigerador para posterior análise. As características morfológicas da superfície do depósito foram analisadas através de microscópio eletrônico de varredura (MEV) e análise química pontual (espectroscopia por energia dispersiva - EDS) utilizando-se aparelho TESCAN, modelo VEGA 3LMH.

Gelatina foi dissolvida no eletrólito antes do início dos experimentos que a incluíram. Foram realizados ensaios variando-se a concentração de gelatina incolor comestível para gerar os depósitos: Depósito D1 não teve adição de gelatina e nos depósitos D2, D3, D4 e D5 houve adição de gelatina com concentrações de $10 \mathrm{mg} / \mathrm{L}, 20 \mathrm{mg} / \mathrm{L}, 40 \mathrm{mg} / \mathrm{L}$ de $80 \mathrm{mg} / \mathrm{L}$, respectivamente, sendo que a densidade de corrente utilizada foi de $342,29 \pm 18,64 \mathrm{~A} / \mathrm{m}^{2}$.

\section{RESULTADOS E DISCUSSÃO}

\subsection{Eficiência de Corrente}

A Tabela 1 apresenta a variação da eficiência de corrente em função da concentração de gelatina. Na solução livre de aditivo, a eficiência de corrente obtida para a deposição de zinco foi de $77,34 \%$. A adição de gelatina em concentração de $10 \mathrm{mg} / \mathrm{L}$ elevou a eficiência de corrente para valores aproximados de $89 \%$. Este aumento na eficiência de corrente é devido, possivelmente, à supressão da evolução de hidrogênio durante a eletrodeposição, por meio do bloqueio de sítios ativos através da adsorção [4]. No entanto, maiores aumentos da concentração de gelatina provocaram queda na eficiência de corrente e, em $80 \mathrm{mg} / \mathrm{L}$, o valor obtido foi de $79 \%$. O valor crítico de concentração de gelatina (concentração na qual a eficiência de corrente começa a diminuir) foi de $20 \mathrm{mg} / \mathrm{L}$.

Tabela 1. Efeitos da gelatina na eficiência de corrente durante eletrodeposição de zinco em solução aquosa de sulfato ácido de zinco na presença e na ausência de gelatina

\begin{tabular}{ccc}
\hline Amostra & Concentração de Gelatina (mg/L) & Eficiência de Corrente (\%) \\
\hline D1 & 0 & 77,34 \\
\hline D2 & 10 & 88,79 \\
\hline D3 & 20 & 85,45 \\
\hline D4 & 40 & 79,85 \\
\hline D5 & 80 & 78,96 \\
\hline
\end{tabular}

\subsection{Morfologia do depósito}

Morfologicamente, foram analisadas as características resultantes da variação da concentração de gelatina no eletrólito com base nas amostras obtidas. Em um primeiro momento, foi realizada uma análise comparativa a olho nu, onde se percebeu que o depósito de zinco obtido da solução de sulfato de zinco livre de aditivo foi 
fracamente brilhante e bastante rugoso (Figura 1). Por outro lado, com a adição de gelatina, os depósitos foram cada vez mais brilhantes e lisos à medida em que se aumentava a concentração da mesma.

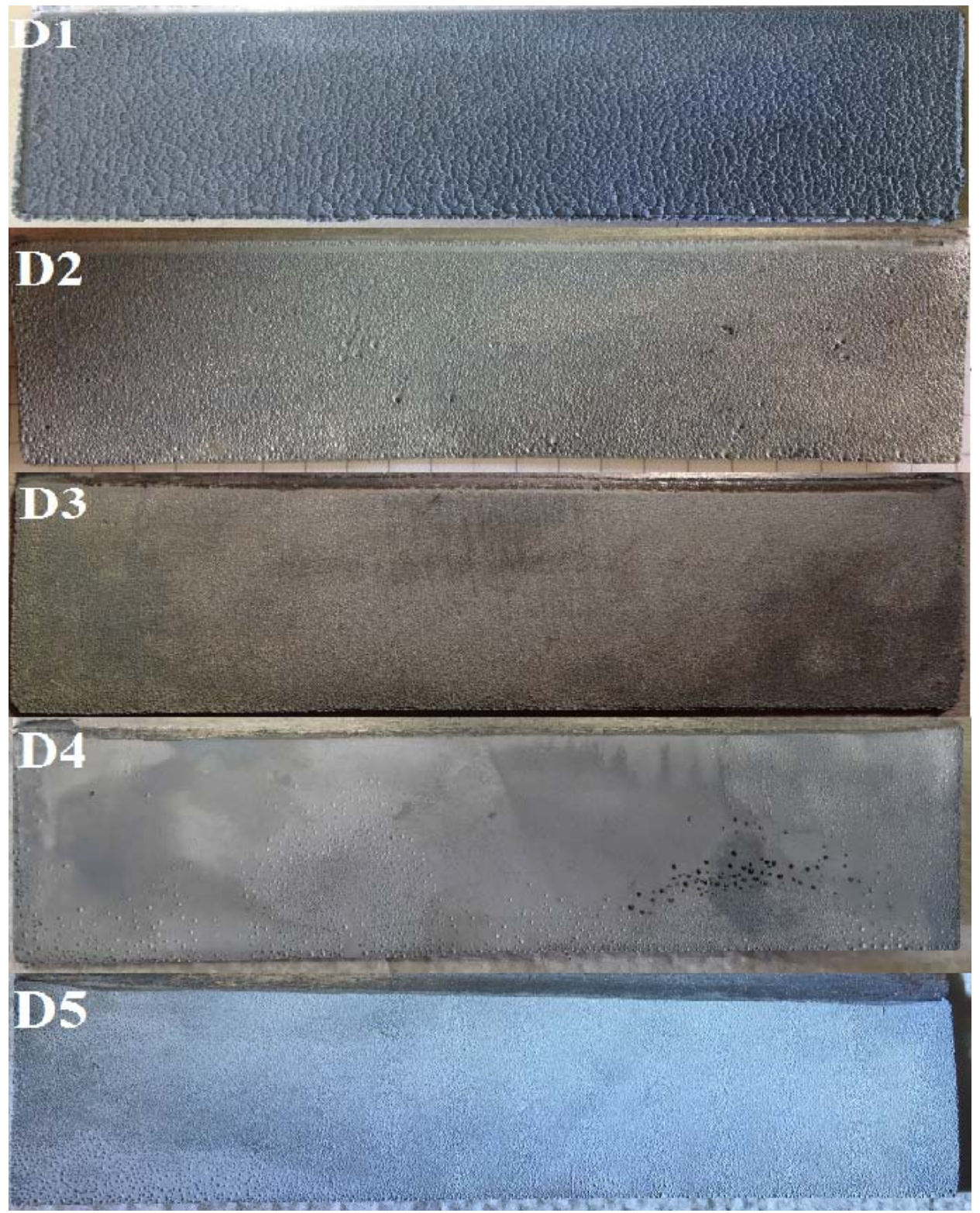

Figura 1. Visão macroscópica (sem aumento) dos depósitos D1 a D5. A solução em que D1 foi obtido não conteve gelatina; nas demais, gelatina estava presente em diferentes concentrações.

Análise em MEV, com aumento de 3000x, revelou que o depósito D1 foi caracterizado por plaquetas hexagonais de tamanhos moderados, sobre as quais foram depositados $\mathrm{ZnSO}_{4} \mathrm{sob}$ a forma de cristais em formato de agulhas (Figura 2). A análise em EDS permitiu identificar resíduos de $\mathrm{Zn}, \mathrm{S}$ e $\mathrm{O}$, confirmando a presença destes elementos no depósito. Ainda para esta solução, foi produzido um depósito de zinco com alta quantidade de poros, resultado de bolhas de hidrogênio aderidas interface Al/Zn que levam à formação e ao crescimento dos mesmos, à medida que o depósito metálico se formou ao redor das bolhas, antes de seu desprendimento. Além disso, a presença de $\mathrm{ZnSO}_{4}$ residual no produto final (depósito) tem implicações práticas, como maior geração de escória na etapa de fundição [3]. 


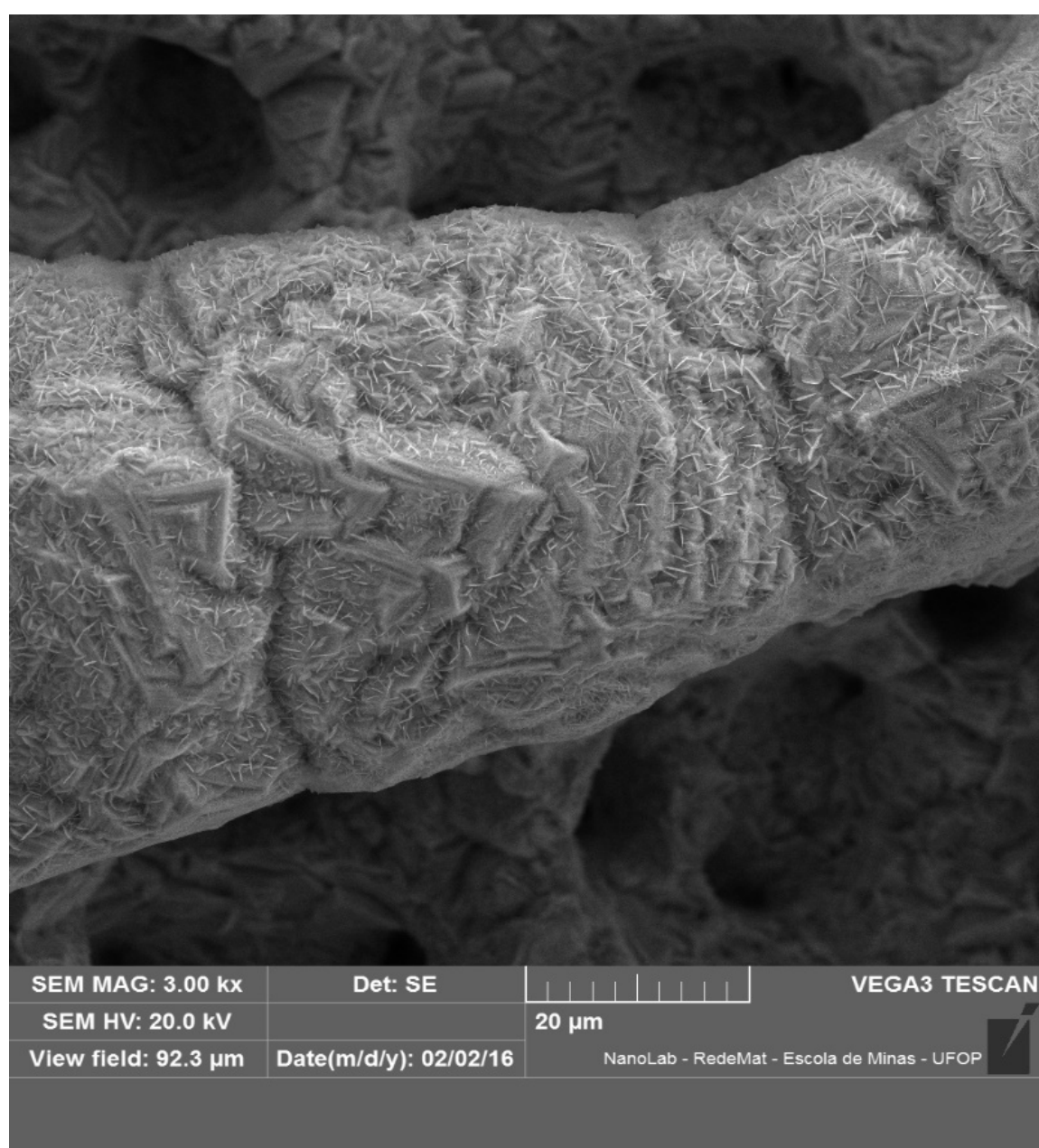

Figura 2. Imagem de D1 em MEV, revelando a presença de agulhas de $\mathrm{ZnSO}_{4}$ depositadas sobre as plaquetas hexagonais de zinco.

Para os depósitos com adição de gelatina, houve um importante aumento na homogeneidade do depósito, com redução do número de poros, proporcionalmente à concentração de gelatina na solução de sulfato de zinco, até a concentração de $40 \mathrm{mg} / \mathrm{L}$, a partir da qual os poros voltaram a ser observados (Figura 3). Este aumento na formação de poros na superfície do depósito pode ser atribuído à adsorção física inicial das moléculas orgânicas de gelatina no catodo Al/Zn [3]. Ademais, o aumento da concentração de gelatina não alterou a morfologia dos cristais de zinco, provocando apenas um refinamento dos grãos, com redução do tamanho das plaquetas hexagonais (Figura 4). 

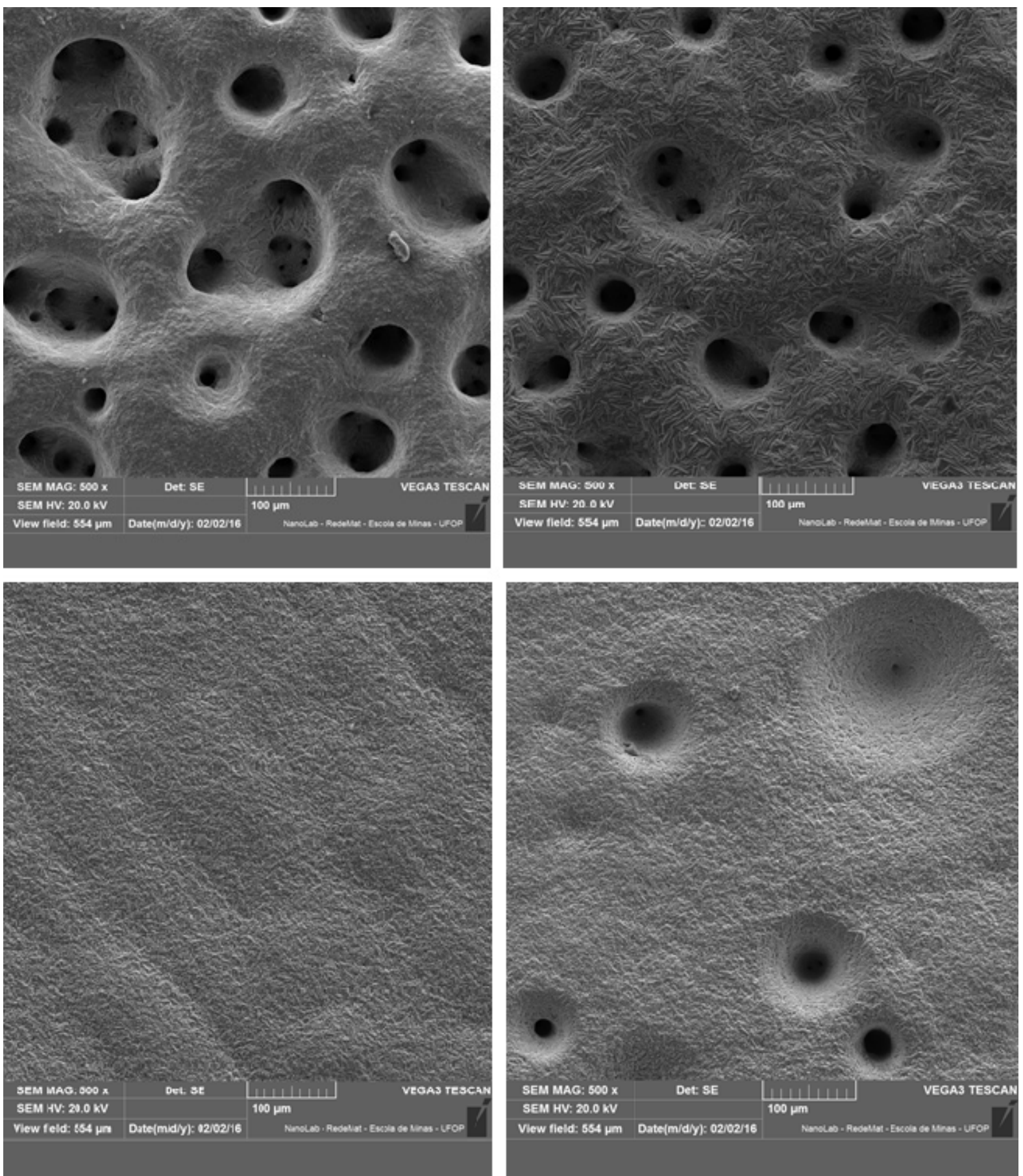

Figura 3. Imagem dos depósitos D2, D3, D4 e D5 obtidos em MEV. Aumento de 500x. Para as concentrações de 10 a $40 \mathrm{mg} / \mathrm{L}$, a quantidade e o tamanho dos poros diminuíram com o aumento da concentração de gelatina na solução. Poros voltaram a aparecer quando a concentração de gelatina foi de $80 \mathrm{mg} / \mathrm{L}$. 

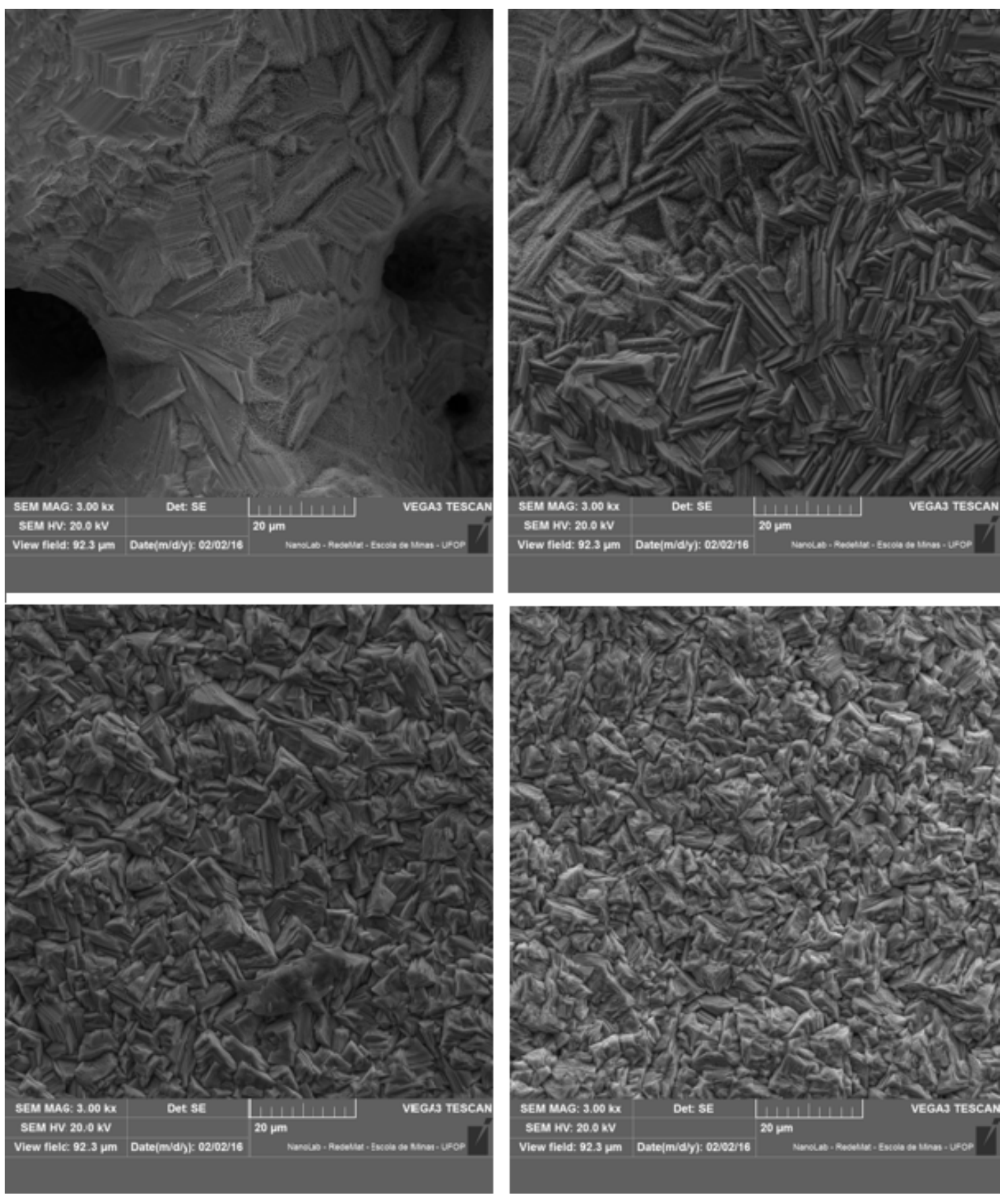

Figura 4. Imagem dos depósitos D2, D3, D4 e D5 obtidos em MEV. Aumento de 3000x. Diminuição do tamanho das plaquetas hexagonais de zinco com o aumento da concentração de gelatina na solução de sulfato de zinco.

\section{CONCLUSÃO}

Em relação à morfologia do depósito, a adição de gelatina produz depósitos de zinco lisos e brilhantes, com melhores morfologias de superfície que em soluções onde a gelatina não esteve presente. A presença de gelatina levou a um depósito com estrutura mais refinada e com uma diminuição da quantidade de poros no depósito de zinco proporcionalmente à sua concentração até o valor de $40 \mathrm{mg} / \mathrm{L}$, de modo que, para a concentração de $80 \mathrm{mg} / \mathrm{L}$ de gelatina, os poros voltaram a aparecer em maiores quantidades. 
A variação na concentração de gelatina não alterou visualmente a forma dos cristais de zinco, contudo, foi observado uma redução do tamanho das plaquetas hexagonais à medida que a concentração de gelatina aumentou, podendo-se afirmar que houve um efeito de refino de grão.

Deve haver uma concentração ótima de gelatina para a qual a eficiência de corrente permanece no intervalo obtido industrialmente (75\% a $90 \%)$, e a quantidade de poros não é fortemente significativa. Este valor, provavelmente, está em torno de $20 \mathrm{mg} / \mathrm{L}$ de gelatina.

Dessa forma, conclui-se que a utilização de gelatina como aditivo em quantidades adequadas pode elevar significativamente a qualidade do depósito de zinco, mas não se sabe sobre a sua interação com outros aditivos comuns na indústria.

\section{Agradecimentos}

Os autores gostariam de agradecer ao Conselho Nacional do Desenvolvimento Científico e Tecnológico (CNPq), à Fundação de Amparo à Pesquisa do Estado de Minas Gerais (FAPEMIG), à Coordenação de Aperfeiçoamento de Pessoal de Nível Superior (CAPES) e à Financiadora de Estudos e Projetos (FINEP) pelo apoio financeiro. A bolsa de pesquisa concedida pela Fundação Gorceix para T. B. Tavares também é especialmente agradecida.

\section{REFERÊNCIAS}

1 Recéndiz A, González I, Nava JL. Current efficiency studies of the zinc electrowinning process on aluminum rotating cylinder electrode (RCE) in sulfuric acid medium: Influence of different additives. Electrochimica Acta. 2007; v. 52 (n. 24): p. 6880-6887.

2 Neves CAR. Zinco. Sumário mineral 2014. Departamento Nacional de Produção Mineral. Brasília, Distrito Federal. 2014. [acesso em 21 fev. 2016]. Disponível em: http://www.dnpm.gov.br/dnpm/sumarios/zinco-sumario-mineral-2014.

3 Majuste D, Martins ELC, Souza AD, Nicol, MJ, Ciminelli VST. Role of organic reagents and impurity in zinc electrowinning. Hydrometallurgy. 2015; v. 152: p. $190-198$.

4 Tripathy BC, Das SC, Singh P, Hefter GT, Misra VN. Zinc electrowinning from acid sulfate solutions Part IV: effects of perfluorocarboxylic acids. Journal of Electroanalytical Chemistry. 2004; v. 565: p. 49-56. 